\title{
Conformal Coupling and Invariance in Different Dimensions
}

\author{
Martin Rainer \\ Projektgruppe Kosmologie, Mathematisches Institut \\ Universität Potsdam, Am Neuen Palais 10 \\ P.O.Box 601553, D-14415 Potsdam, Germany \\ and \\ Gravitationsprojekt, Universität Potsdam \\ An der Sternwarte 16 \\ D-14482 Potsdam, Germany
}

\begin{abstract}
Conformal transformations of the following kinds are compared: (1) conformal coordinate transformations, (2) conformal transformations of Lagrangian models for a $D$-dimensional geometry, given by a Riemannian manifold $M$ with metric $g$ of arbitrary signature, and (3) conformal transformations of (mini-)superspace geometry. For conformal invariance under this transformations the following applications are given respectively: (1) Natural time gauges for multidimensional geometry, (2) conformally equivalent Lagrangian models for geometry coupled to a spacially homogeneous scalar field, and (3) the conformal Laplace operator on the $n$-dimensional manifold $\mathcal{M}$ of minisuperspace for multidimensional geometry and the Wheeler de Witt equation.

The conformal coupling constant $\xi_{c}$ is critically distinguished among arbitrary couplings $\xi$, for both, the equivalence of Lagrangian models with $D$-dimensional geometry and the conformal geometry on $n$-dimensional minisuperspace.

For dimension $D=3,4,6$ or 10 , the critical number $\xi_{c}=\frac{D-2}{4(D-1)}$ is especially simple as a rational fraction.
\end{abstract}

PACS No.: 0460, 0240 


\section{Introduction}

Recently conformal transformations between different multidimensional geometrical models ${ }^{1-3}$ and the question of conformal equivalence receive increasing interest for cosmology. While cosmologist in recent time apply conformal transformations already to minisuperspace and the Wheeler de Witt (WdW) equation, several mathematical questions concerning the structure of such transformations are, besides lacking mathematical clarification of the (mini-)superspace construction, still open, although Refs. 4 and 5 indicate some progress. In Ref. 6 (and also Refs. therein) conformal transformations have already been discussed systematically within both, the class of higher order gravity and that of gravity coupled to a scalarfield, and between these two classes. This paper is intended to contibute to an understanding of conformal transformations of minisuperspace geometry as compared to conformal transformations of ordinary geometry and coordinate transformations. The conformal coupling constant $\xi_{c}$ will play a distinguished role for conformal invariance in different contexts like equivalent Lagrangian models and the conformal Laplace operator. Furthermore its dependence on the dimension of the underlying space has interesting number theoretical properties, distinguishing those low dimensions which appear in prefered theories of the universe.

In Sec. 2 we compare conformal transformations of the following kinds: (1) conformal coordinate transformations, (2) conformal transformations of Lagrangian models for a $D$ dimensional geometry, given by a Riemannian manifold $M$ with metric $g$ of arbitrary signature, and (3) conformal transformations of (mini-)superspace geometry.

As application of invariance under (1), in Sec. 3 special emphasis is put to compare natural time gauges for multidimensional universes given by the choices of $i$ ) the synchronous time $t_{s}$ of the universe $M$, ii) the conformal time $\eta_{i}$ of a universe with the only spacial factor $M_{i}$, iii) the mean conformal time $\eta$, given differentially as some scale factor weighted average of $\eta_{i}$ over all $i$ and iv) the harmonic time $t_{h}$, which will be used as specially convenient in calculations on minisuperspace, since in this gauge the minisuperspace lapse function is $N \equiv 1$.

As application of invariance under (2) in Sec. 4 we examine conformal transformations between conformally equivalent Lagrangian models for $D$-dimensional geometry coupled to a spacially homogeneous scalar field. The conformal coupling const $\xi_{c}$ here plays a distinguished role. We consider as example of special interest the conformal transformation 
between a model with minimally coupled scalar field and an equivalent conformal model with a conformally coupled scalar field, thus generalizing previous results from Refs. 7, 8 and 9 for $D=4$.

In Sec. 5 we derive the unique conformal Laplace(-Beltrami) operator on a (Pseudo) Riemannian manifold $\mathcal{M}$ of dimension $n$. Though this had been given already by construction of a conformal WdW equation in Ref. 10, and in the mathematical literature there is agreement on a linear coupling $\Delta+a R$ of Laplacian $\Delta$, generalized from the flat case, and Ricci curvature scalar $R$ on the underlying manifold, there is sometimes some confusion ${ }^{11}$ about the proper choice of the coupling $a$ on an arbitrarily curved manifold. Therefore here we prove that $\Delta+a R$ is conformal if and only if $n>1$ and $a=-\xi_{c}$.

As application of equivariance w.r.t. tranformations (3) in Sec. 6 we motivate a minisuperspace for multidimensional geometry with minimally coupled scalar field, and get a first quantization of the energy constraint to the WdW equation in a both generally covariant and conformally equivariant manner, where the Laplace operator of Sec. 5 is essential ingredient.

Sec. 7 is devoted to a number theoretical examination of the rational number $\xi_{c}=$ $\frac{D-2}{4(D-1)}$, revealing distinguished dimensions $D=3,4,6$ and 10 .

Sec. 8 resumes the perspective of the present results.

\section{Conformal Transformations}

Generally we will have to distinguish between (1) conformal coordinate transformations in $D$-dimensional geometry (2) conformal transformations of $D$-dimensional geometry, especially in Lagrangian models, and (3) conformal transformations of $n$-dimensional minisuperspace geometry.

\section{(1) Conformal transformation to new coordinates:}

We fix the geometry and transform the metric tensor components conformally,

$$
g_{i^{\prime} j^{\prime}}=e^{2 f(x)} g_{k l}
$$

via a coordinate transform satisfying

$$
d x^{\prime i}=e^{-f(x)} d x^{i} \quad \text { or } \quad \frac{\partial x^{i}}{\partial x^{j}}=e^{-f(x)} \delta_{j}^{i} .
$$


Here the first fundamental form

$$
d s^{2}=g_{i^{\prime} j^{\prime}} d x^{i^{\prime}} d x^{j^{\prime}}=g_{i j} d x^{i} d x^{j},
$$

and therefore the (inner) geometry, remains invariant, though looking different in different coordinate frames.

(2) Conformal transformations of ordinary ( $D$-dimensional) geometry:

Let us consider a differentiable manifold $M$. On a (Pseudo-)Riemannian geometry $g$ on $M$, conformal transformations will be represented as Weyl transformations $g \mapsto e^{2 f} g$ with $f \in$ $C^{\infty}(M)$. For a structure on $M$ given by the metric components $g_{i j}$ and (additional) scalar fields $\left(\phi^{1}, \ldots, \phi^{k}\right)$, a second order Lagrangian model consists in a Lagrangian variation principle

$$
\delta S=0 \quad \text { with } \quad S=\int_{M} \sqrt{|g|} L d^{D} x
$$

with Lagrangian

$$
L=L\left(g_{i j}, \phi^{1}, \ldots, \phi^{k} ; g_{i j, l}, \phi_{, l}^{1}, \ldots, \phi_{, l}^{k} ; g_{i j, l m}\right) .
$$

Conformal transformation of the Lagrangian model keeps $M$ fixed as a differentiable manifold, but varies its additional structures conformally

$$
\left(g_{i j}, \phi^{1}, \ldots, \phi^{k}\right) \rightarrow\left(\hat{g}_{i j}, \hat{\phi}^{1}, \ldots, \hat{\phi}^{k}\right),
$$

yielding a new variational principle by demanding

$$
\sqrt{|g|} L \stackrel{!}{=} \sqrt{|\hat{g}|} \hat{L}
$$

for the new Lagrangian

$$
\hat{L}=\hat{L}\left(\hat{g}_{i j}, \hat{\phi}^{1}, \ldots, \hat{\phi}^{k} ; \hat{g}_{i j, l}, \hat{\phi}_{, l}^{1}, \ldots, \hat{\phi}_{, l}^{k} ; \hat{g}_{i j, l m}\right) .
$$

Therefore conformal transformations of (Lagrangian models for) geometry (plus eventual scalar fields) are performed in practice on a fixed coordinate patch $x^{i}$ of $M$.

(3) Conformal transformation of minisuperspace geometry:

In Ref. 5 superspace is defined as the geometries $\operatorname{Met}(M) / \operatorname{Diff}(M)$. We will not consider the here the difficult question of $\operatorname{Diff}(M)$-equivalence. Let us assume that this is solved by a kind of general coordinate invariance of superspace. In Ref. 4 it was shown how the space of Riemannian metrics $\operatorname{Met}(M)$ can be equipped with a metric $G$. This is what we will 
do here. Let us pick some superspace coordinates $\chi^{A}$ indexed by $A$ within an appropriate index set. Then we consider

$$
G=G_{A B} d \chi^{A} \otimes d \chi^{B}
$$

as defined via

$$
G_{A B}:=G_{i j k l} h_{A}^{i j} h_{B}^{k l}
$$

where

$$
G_{i j k l}:=g_{i k} g_{j l}+g_{i l} g_{j k}-g_{i j} g_{k l} .
$$

Note the similarity of Eq. (2.11) to the usual first Christoffel symbols. Both $G_{i j k l}$ and $h_{A}^{i j}$ are covariant 4- resp. 2-tensor components with respect to usual coordinate transforms. Therefore $G_{A B}$ is independent of coordinates on $M$.

The components $h_{A}^{i j}$ define a generalized soldering form $\theta:=h_{A}^{i j} e_{i j} \otimes d \chi^{A}$, where 2tensors $e_{i j}=h_{i j}^{A} \frac{\partial}{\partial \chi^{A}}$ are given by components $h_{i j}^{A}$ dual to $h_{A}^{i j}$.

Eq. (2.11) singles out a special class of supermetrics, and, together with general superspace covariance, yields a reduced superspace. However without further restriction its index set would be still infinite.

A further reduction of superspace to yielding finite dimensions is is well defined for a class of metrics of multidimensional type. Here a geometry is described on a (Pseudo-) Riemannian manifold

$$
\begin{gathered}
M=\mathbb{R} \times M_{1} \times \ldots \times M_{n}, \quad D:=\operatorname{dim} M=1+d_{1}+\ldots+d_{n}, \\
g \equiv d s^{2}=-e^{2 \gamma} d t \otimes d t+\sum_{i=1}^{n} a_{i}^{2} d s_{i}^{2}
\end{gathered}
$$

where $a_{i}=e^{\beta^{i}}$ is the scale factor of the $d_{i}$-dimensional space $M_{i}$ with the first fundamental form

$$
d s_{i}^{2}=g_{k l}^{(i)} d x_{(i)}^{k} \otimes d x_{(i)}^{l} .
$$

Then the scalefactors $e^{\beta^{A}}, A=1, \ldots, n$, yield (reduced) supercoordinates

$$
\chi^{A}:=e^{2 \beta^{A}}, \quad A=1, \ldots, n .
$$

The minisuperspace $\mathcal{M S}(M)$ over $M$ is then defined by minisuperspace coordinates $\beta^{1}, \ldots, \beta^{n}$ subject to the principle of general covariance w.r.t. minisuperspace coordinate transformations. 
Invariance of (2.10) under conformal transformations (2) with $g \mapsto e^{2 f} g$ yields invariance under

$$
h_{A}^{i j} \mapsto e^{-2 f} h_{A}^{i j},
$$

which corresponds to invariance under supercoordinate transformations

$$
\chi^{A} \mapsto e^{2 f} \chi^{A}
$$

The conformal weight differs from that of an analogous ordinary coordinate transformation (2.2) by a factor -2 , corresponding to the fact that $h_{A}^{i j}$ contragrediently relates supervectors to 2 -tensors.

For a minisuperspace $\mathcal{M}=\mathcal{M S}(M)$ from (2.13) the supercoordinate tranformations (2.15) correspond via (2.13) to translations of the minisuperspace coordinates

$$
\beta^{i} \rightarrow \beta^{i}+f
$$

So conformal transformations (2) of multidimensional geometry $M$ yield just supercoordinate transformations in $\mathcal{M}$.

Well distinguished from the latter, conformal transformations of the minisuperspace geometry

$$
G=G_{i j} d \beta^{i} \otimes d \beta^{j}
$$

are given by

$$
G \mapsto{ }^{f} G:=e^{2 f} G
$$

with $f \in C^{\infty}(\mathcal{M})$.

So far we demonstrated the necessity to distinguish transformations (3) against (2), in analogy to the difference between transformations (2) and (1).

Applications of invariance under transformations (2) and (3) will be given later. A special application of transformations (1) are time gauge transformations, from arbitrarily given coordinates to one of the natural time gauges considered in the following section. 


\section{$3 \quad$ Natural Times in Multidimensional Geometry}

Let us consider a multidimensional geometry like in Eq. (2.12) and compare different choices of time $t$ in Eq. (2.12). The time gauge is determined by the function $\gamma$. There exist few natural time gauges from the physical point of view.

i) The synchronous time gauge

$$
\gamma \equiv 0
$$

for which $t$ in Eq. (2.12) is the proper time $t_{s}$ of the universe. The clocks of geodesically comoved observers go synchronous to that time.

ii) The conformal time gauges on $\mathbb{R} \times M_{i} \subset M$

$$
\gamma \equiv \beta^{i},
$$

for which $t$ in Eq. (2.12) is the conformal time $\eta_{i}$ of $M_{i}$ for some $i \in\{1, \ldots, n\}$, given by

$$
d \eta_{i}=e^{-\beta^{i}} d t_{s}
$$

iii) The mean conformal time gauge on $M$ :

For $n>1$ and $\beta^{2} \neq \beta^{1}$ on $M$ the usual concept of a conformal time does no longer apply. Looking for a generalized "conformal time" $\eta$ on $M$, we set

$$
d:=D-1=\sum_{i=1}^{n} d_{i}
$$

and consider the gauge

$$
\gamma \equiv \frac{1}{d} \sum_{i=1}^{n} d_{i} \beta^{i}
$$

which yields a time $t \equiv \eta$ given by

$$
d \eta=\left(\prod_{i=1}^{n} a_{i}^{d_{i}}\right)^{-1 / d} d t_{s}
$$

Here $\prod_{i=1}^{n} a_{i}^{d_{i}}$ is proportional to the volume of $d$-dimensional spacial sections in $M$ and the relative time scale factor

$$
\left(\prod_{i=1}^{n} a_{i}^{d_{i}}\right)^{1 / d}=e^{\frac{1}{d} \sum_{i} d_{i} \beta^{i}}
$$

is given by a scale exponent, which is the dimensionally weighted arithmetic mean of the spacial scaling exponents of spaces $M_{i}$. It is

$$
\left(d t_{s}\right)^{d}=e^{\sum_{i} d_{i} \beta^{i}} d \eta^{d} .
$$


Since on the other hand by Eq. (3.3) we have

$$
\left(d t_{s}\right)^{d}=\otimes_{i=1}^{n}\left(e^{\beta^{i}} d \eta_{i}\right)^{d_{i}}
$$

together with Eq. (3.8) we yield

$$
(d \eta)^{d}=e^{-\sum_{i} d_{i} \beta^{i}} \otimes_{i=1}^{n}\left(e^{\beta^{i}} d \eta_{i}\right)^{d_{i}} .
$$

So the time $\eta$ is a mean conformal time, given differentially as a dimensionally scale factor weighted geometrical tensor average of the conformal times $\eta_{i}$. An alternative to the mean conformal time $\eta$ is given by a similar differential averaging like Eq. (3.10), but weighted by an additional factor of $e^{(1-d) \sum_{i} d_{i} \beta^{i}}$. This is gauge is described in the following.

iv) The harmonic time gauge

$$
\gamma \equiv \gamma_{h}:=\sum_{i=1}^{n} d_{i} \beta^{i}
$$

yields the time $t \equiv t_{h}$, given by

$$
d t_{h}=\left(\prod_{i=1}^{n} a_{i}^{d_{i}}\right)^{-1} d t_{s}=\left(\prod_{i=1}^{n} a_{i}^{d_{i}}\right)^{\frac{1-d}{d}} d \eta .
$$

In this gauge any function $\varphi$ with $\varphi(t, y)=t$ is harmonic, i.e. $\Delta[g] \varphi=0$, and the minisuperspace lapse function is $N \equiv 1$. The latter is especially convenient when we work in minisuperspace.

\section{Conformally Equivalent Lagrangian Models}

Now we want to study the effect of transformations (2) in more detail. One application of special interest is the transformation from a Lagrangian model with minimally coupled scalarfield to a conformally equivalent one with non-minimally coupled scalarfield and vice versa.

Let us follow Ref. 12 and consider an action of the kind

$$
S=\int d^{D} x \sqrt{|g|}\left(F(\phi, R)-\frac{\epsilon}{2}(\nabla \phi)^{2}\right) .
$$

With

$$
\omega:=\frac{1}{D-2} \ln \left(2 \kappa^{2}\left|\frac{\partial F}{\partial R}\right|\right)+C
$$


the conformal factor

$$
e^{\omega}=\left[2 \kappa^{2}\left|\frac{\partial F}{\partial R}\right|\right]^{\frac{1}{D-2}} e^{C}
$$

yields a conformal transformation from $g_{\mu \nu}$ to the minimally coupled metric

$$
\hat{g}_{\mu \nu}=e^{2 \omega} g_{\mu \nu}
$$

Especially let us consider in the following actions, which are linear in $R$. With

$$
F(\phi, R)=f(\phi) R-V(\phi)
$$

the action is

$$
S=\int d^{D} x \sqrt{|g|}\left(f(\phi) R-V(\phi)-\frac{\epsilon}{2}(\nabla \phi)^{2}\right) .
$$

The minimal coupling metric is then related to the conformal one by (4.4) with

$$
\omega=\frac{1}{D-2} \ln \left(2 \kappa^{2}|f(\phi)|\right)+C
$$

The scalar field in the minimal coupling model is

$$
\begin{gathered}
\Phi=\kappa^{-1} \int d \phi\left\{\frac{\epsilon(D-2) f(\phi)+2(D-1)\left(f^{\prime}(\phi)\right)^{2}}{2(D-2) f^{2}(\phi)}\right\}^{1 / 2}= \\
=(2 \kappa)^{-1} \int d \phi\left\{\frac{2 \epsilon f(\phi)+\xi_{c}^{-1}\left(f^{\prime}(\phi)\right)^{2}}{f^{2}(\phi)}\right\}^{1 / 2},
\end{gathered}
$$

where

$$
\xi_{c}:=\frac{D-2}{4(D-1)}
$$

is the conformal coupling constant.

For the following we define $\operatorname{sign} x$ to be \pm 1 for $x \geq 0$ resp. $x<0$. Then with the new minimal coupling potential

$$
U(\Phi)=(\operatorname{sign} f(\phi))\left[2 \kappa^{2}|f(\phi)|\right]^{-D / D-2} V(\phi)
$$

the corresponding minimal coupling action is

$$
S=\operatorname{sign} f \int d^{D} x \sqrt{|\hat{g}|}\left(-\frac{1}{2}\left[(\hat{\nabla} \Phi)^{2}-\frac{1}{\kappa^{2}} \hat{R}\right]-U(\Phi)\right) .
$$

Example 1:

$$
f(\phi)=\frac{1}{2} \xi \phi^{2}
$$




$$
V(\phi)=-\lambda \phi^{\frac{2 D}{D-2}}
$$

Substituting this into Eq. (4.10) the corresponding minimal coupling potential $U$ is constant,

$$
U(\Phi)=(\operatorname{sign} \xi)\left|\xi \kappa^{2}\right|^{-D / D-2} \lambda
$$

It becomes zero precisely for $\lambda=0$, i.e. when $V$ is zero. With

$$
f^{\prime}(\phi)=\xi \phi
$$

we obtain

$$
\begin{gathered}
\Phi=\kappa^{-1} \int d \phi\left\{\frac{\left(\frac{\epsilon}{\xi}+\frac{1}{\xi_{c}}\right) \phi^{2}}{\phi^{4}}\right\}^{\frac{1}{2}}=(\kappa \sqrt{\xi})^{-1} \sqrt{\frac{1}{\xi_{c}}+\frac{\epsilon}{\xi}} \int d \phi \frac{1}{|\phi|} \\
=\kappa^{-1} \sqrt{\frac{1}{\xi_{c}}+\frac{\epsilon}{\xi}} \ln |\phi|+C
\end{gathered}
$$

for $-\frac{\xi}{\epsilon} \geq \xi_{c}$. Note that for

$$
\frac{\xi}{\epsilon}=-\xi_{c}
$$

e.g. for $\epsilon=-1$ and conformal coupling, we have

$$
\Phi=C
$$

Thus here the conformal coupling theory is equivalent to a theory without scalarfield.

For $-\frac{\xi}{\epsilon}<\xi_{c}$ the field $\Phi$ would become complex and, for imaginary $C$, purely imaginary.

In any case the integration constant $C$ may be a function of the coupling $\xi$ and the dimension $D$.

Example 2:

$$
\begin{gathered}
f(\phi)=\frac{1}{2}\left(1-\xi \phi^{2}\right), \\
V(\phi)=\Lambda .
\end{gathered}
$$

Then the constant potential $V$ has its minimal coupling correspondence in a non constant $U$, given by

$$
U(\Phi)= \pm \Lambda\left|\kappa^{2}\left(1-\xi \phi^{2}\right)\right|^{-D / D-2}
$$

respectively for $\phi^{2}<\xi^{-1}$ or $\phi^{2}>\xi^{-1}$.

Let us set in the following

$$
\epsilon=1
$$


Then with

$$
f^{\prime}(\phi)=-\xi \phi
$$

we obtain

$$
\Phi=\kappa^{-1} \int d \phi\left\{\frac{1+c \xi \phi^{2}}{\left(1-\xi \phi^{2}\right)^{2}}\right\}^{1 / 2}
$$

where

$$
c:=\frac{\xi}{\xi_{c}}-1
$$

For $\xi=0$ it is $\Phi=\kappa^{-1} \phi+A$, i.e. the coupling remains minimal.

To solved this integral for $\xi \neq 0$, we substitute $u:=\xi \phi^{2}$.

To assure a solution of (4.24) to be real, let us assume $\xi \geq \xi_{c}$ which yields $c \geq 0$.

Then we obtain

$$
\begin{gathered}
\Phi=\frac{\operatorname{sign}(\phi)}{2 \kappa \sqrt{\xi}} \int \frac{\sqrt{u^{-1}+c}}{|1-u|} d u+C_{>} \\
=\frac{\operatorname{sign}((1-u) \phi)}{2 \kappa \sqrt{\xi}}[-\sqrt{c} \ln (2 \sqrt{c} \sqrt{1+c u} \sqrt{u}+2 c u+1)+ \\
\left.\sqrt{1+c} \ln \left(\frac{2 \sqrt{1+c} \sqrt{1+c u} \sqrt{u}+2 c u+1+u}{|1-u|}\right)\right]+C_{>} \\
=\frac{\operatorname{sign}\left(\left(1-\xi \phi^{2}\right) \phi\right)}{2 \kappa \sqrt{\xi}}\left\{-\sqrt{c} \ln \left(2 \sqrt{c} \sqrt{1+c \xi \phi^{2}} \sqrt{\xi}|\phi|+2 c \xi \phi^{2}+1\right)\right. \\
\left.+\sqrt{1+c} \ln \left(\frac{2 \sqrt{1+c} \sqrt{1+c \xi \phi^{2}} \sqrt{\xi}|\phi|+2 c \xi \phi^{2}+1+\xi \phi^{2}}{\left|1-\xi \phi^{2}\right|}\right)\right\}+C_{>} \\
=\frac{\operatorname{sign}\left(\left(1-\xi \phi^{2}\right) \phi\right)}{2 \kappa \sqrt{\xi}} \ln \frac{\left[2 \sqrt{1+c} \sqrt{1+c \xi \phi^{2}} \sqrt{\xi}|\phi|+(2 c+1) \xi \phi^{2}+1\right]^{\sqrt{1+c}}}{\left[2 \sqrt{c} \sqrt{1+c \xi \phi^{2}} \sqrt{\xi}|\phi|+2 c \xi \phi^{2}+1\right]^{\sqrt{c}} \cdot\left|1-\xi \phi^{2}\right|^{\sqrt{1+c}}} \\
+C_{<} .
\end{gathered}
$$

The integration constants $C<$ for $\phi^{2}<\xi^{-1}$ and $\phi^{2}>\xi^{-1}$ respectively may be arbitrary functions of $\xi$ and the dimension $D$.

The singularities of the transform $\phi \rightarrow \Phi$ are located at $\phi^{2}=\xi^{-1}$. An expression corresponding to (4.26) with $D=4$ qualitatively has already been given in Ref. 7 .

If the coupling is conformal $\xi=\xi_{c}$, i.e. $c=0$, the expressions (4.26) simplify to

$$
\kappa \Phi=\frac{1}{\sqrt{\xi_{c}}}\left[\left(\operatorname{artanh} \sqrt{\xi_{c}} \phi\right)+c_{<}\right]
$$

for $\phi^{2}<\xi_{c}^{-1}$ and to

$$
\kappa \Phi=\frac{1}{\sqrt{\xi_{c}}}\left[\left(\operatorname{arcoth} \sqrt{\xi_{c}} \phi\right)+c_{>}\right]
$$


for $\phi^{2}>\xi_{c}^{-1}$.

Then the inverse formulas expressing the conformal field $\phi$ in terms of the minimal coupling field $\Phi$ are

$$
\phi=\frac{1}{\sqrt{\xi_{c}}}\left[\tanh \left(\sqrt{\xi_{c}} \kappa \Phi-c_{<}\right)\right]
$$

with $\phi^{2}<\xi_{c}^{-1}$ and

$$
\phi=\frac{1}{\sqrt{\xi_{c}}}\left[\left(\operatorname{coth}\left(\sqrt{\xi_{c}} \kappa \Phi-c_{>}\right)\right]\right.
$$

with $\phi^{2}>\xi_{c}^{-1}$ respectively. This result agrees with Ref. 13. For $D=4$ it has been obtained earlier in Refs. 8, 9 and 14. In Ref. 14 it has been shown for $D=4$, that while the minimal coupling model shows a curvature singularity, the conformal coupling model with $\phi$ of Eq. (4.29) has no such singularity.

The conformal factor is according to Eqs. (4.7) and (4.19) given by

$$
\omega=\frac{1}{D-2} \ln \left(\kappa^{2}\left|1-\xi_{c} \phi^{2}\right|\right)+C .
$$

The singularity of the conformal transformation (4.31) at $\phi^{2}=\xi_{c}^{-1}$ separates different regions in $\phi$ where conformal equivalence between the minimal and conformal coupling model holds. Eqs. (4.29) and (4.30) illustrate the qualitatively different behavior in the two regions. In Ref. 1 this qualitative difference have also been found in multidimensional solutions of the respective models.

Note finally that, if e.g. time is harmonic in the minimal coupling model

$$
\tau \equiv t_{h}^{(m)},
$$

in the conformal model it cannot be expected to be harmonic either, i.e. in general

$$
\tau \neq t_{h}^{(c)} .
$$

Natural time gauges are not preserved by conformal transformations (2). Usually they have to be calculated by a coordinate transformation in each of the equivalent models separately.

\section{The Conformal Laplace Operator}

In this section we search for a linear combination

$$
\Delta_{a}=\Delta+a R
$$


of the Laplace-Beltrami operator $\Delta=\Delta[G]$ and the Ricci scalar curvature $R=R[G]$ of an $n$-dimensional manifold $\mathcal{M}$, such that $\Delta_{a}$ is not only a generally covariant but also a conformal operator of weight -2 , which furthermore transforms according to the conjugate representation $D_{b}$ of Weyl transformations of weight $b$ on the Hilbertspace $\mathcal{H}(\mathcal{M})$. With $f \in C^{\infty}(M)$, the latter transform $\mathcal{H}:=\mathcal{H}(\mathcal{M})$ to $\mathcal{H}^{f}:=e^{b f} \mathcal{H}$. Then the conformal operator on $\mathcal{H}^{f}$ is

$$
\Delta_{a}^{f}=e^{(b-2) f} \Delta_{a} e^{-b f}
$$

where $\Delta_{a}^{f}=\Delta^{f}+a R^{f}$ with

$$
\Delta^{f}={ }^{f} G^{i j} \nabla_{i}^{f} \nabla_{j}^{f}
$$

Here the covariant derivative $\nabla^{f}$ is determined by the connection $\Gamma^{f}$ w.r.t. the metric $G^{f}$. Since the components of the inverse metric are

$$
{ }^{f} G^{i j}=e^{-2 f} G^{i j}
$$

the connection coefficients are

$$
\begin{gathered}
{ }_{\Gamma_{i j}}^{k}=\frac{1}{2}{ }^{f} G^{k l}\left\{{ }^{f} G_{l i, j}+{ }^{f} G_{l i, j}-{ }^{f} G_{i j, l}\right\} \\
=\Gamma_{i j}^{k}+\left\{\delta_{i}^{k} f_{, j}+\delta_{j}^{k} f_{, i}-G_{i j} f, k\right\}
\end{gathered}
$$

and the Ricci scalar w.r.t. ${ }^{f} G$ is

$$
\begin{gathered}
{ }^{f} R=e^{-2 f} G^{c d}\left[R_{c d}-2(n-1) f_{, c d}-(n-1)(n-2) f_{, c} f_{, d}+2(n-1) \Gamma_{c d}^{e} f_{, e}\right], \\
=e^{-2 f}\left\{R-2(n-1) \Delta f-(n-1)(n-2) f^{, k} f_{, k}\right\} .
\end{gathered}
$$

On $\mathcal{H}^{f}$ we find

$$
\begin{gathered}
\Delta^{f}={ }^{f} G^{i j} \nabla_{i} \partial_{j} \\
=e^{-2 f} \Delta-e^{-2 f} G^{c d}\left\{f_{, i} \partial_{j}+f_{, j} \partial_{i}-\Gamma_{i j} f^{k} \partial_{k}\right\}
\end{gathered}
$$

in terms of the original metric $G$ and its Laplacian $\Delta$ on acting on $\mathcal{H}$. Thus we obtain

$$
\Delta^{f} \Psi^{f}=e^{(b-2) f}\left\{\Delta \Psi+[2(b-1)+n] f^{, k} \Psi_{, k}+\left[b \Delta f+(b+n-2) b f^{k} f_{k}\right] \Psi\right\}
$$

and together with Eq. (5.6) it is

$$
\Delta_{a}^{f} \Psi^{f}=e^{(b-2) f}\left\{\Delta_{a} \Psi+[2(b-1)+n] f^{, k} \Psi_{, k}+\left[A \Delta f+B f^{k} f_{k}\right] \Psi\right\}
$$


with coefficients

$$
A=b-2(n-1) a \text { and } B=(b+n-2) b-(n-1)(n-2) a .
$$

Vanishing of the $f^{, k} \Psi_{, k}$ term in Eq. (5.9) requires

$$
b=1-\frac{n}{2}=-\frac{n-2}{2},
$$

which then yields the coefficients

$$
A=-\frac{1}{2}\{4(n-1) a+(n-2)\}
$$

and

$$
B=-\frac{n-2}{4}\{4(n-1) a+(n-2)\}
$$

both proportional to $4(n-1) a+(n-2)$. Then for $n \neq 1$ their vanishing requires

$$
a=-\frac{n-2}{4(n-1)} \equiv-\xi_{c}
$$

For $n=1$ the condition (5.11) implies $b=\frac{1}{2}$ where $A \neq 0 \neq B$, while vanishing of $A$ and $B$ according to Eq. (5.10) implies $b=0$ where condition (5.11) is violated. Thus for $n=1$ there is no conformal operator (5.1) for any value of $a$. This is because every 1-dimensional manifold is intrinsically flat and hence $R \equiv 0$, while the representation $D_{b}$ is non trivial for $b=\frac{1}{2}$.

Note that condition (5.11) excludes the trivial representation $b=0$ in all dimensions except $n=2$, where $a=b=0$ and $\Psi^{f}=\Psi$ for all $f$, since any 2-dimensional manifold is conformally flat.

We find that the operator (5.1) is conformally invariant if and only if $n \neq 1$ and the values of $a$ and $b$ are given by Eq. (5.11) and (5.14).

\section{Minisuperspace and the $\mathrm{WdW}$ Equation}

If we assume within a multidimensional geometry (2.12) that $M_{i}$ are Einstein spaces, they satisfy the equations

$$
R_{k l}^{(i)}=\lambda_{i} g_{k l}^{(i)},
$$

and hence

$$
R^{(i)}=\lambda_{i} d_{i}
$$


Here the Ricci tensor and scalar are defined as usual by

$$
R_{\mu \nu}:=R_{\mu \lambda \nu}^{\lambda} \quad \text { and } \quad R:=R_{\mu}^{\mu} .
$$

If furthermore $M_{i}$ is of constant curvature, then

$$
d s_{i}^{2}=\frac{1}{\left(1+\frac{1}{4} K_{i} r_{i}^{2}\right)^{2}} \sum_{k=1}^{d_{i}} d x_{(i)}^{k} \otimes d x_{(i)}^{k},
$$

with radial variable $r_{i}=\sqrt{\sum_{k=1}^{d_{i}}\left(x_{(i)}^{k}\right)^{2}}$ and constant sectional curvature, normalized with $K_{i}= \pm 1$ for positive and negative $K_{i}$ respectively. In the flat case $K_{i}=0$. Then the Riemann tensor of $M_{i}$ is

$$
R_{k l m n}^{(i)}=K_{i}\left(g_{k m}^{(i)} g_{l n}^{(i)}-g_{k n}^{(i)} g_{l m}^{(i)}\right)
$$

Ricci tensor and scalar are then given by Eq. (6.1) and (6.2) with

$$
\lambda_{i} \equiv K_{i}\left(d_{i}-1\right) .
$$

For the geometry (2.12) the Ricci scalar curvature of $M$ is

$$
R=e^{-2 \gamma}\left\{\left[\sum_{i=1}^{n}\left(d_{i} \dot{\beta}^{i}\right)\right]^{2}+\sum_{i=1}^{n} d_{i}\left[\left(\dot{\beta}^{i}\right)^{2}-2 \dot{\gamma} \dot{\beta}^{i}+2 \ddot{\beta}\right]\right\}+\sum_{i=1}^{n} R^{(i)} e^{-2 \beta^{i}} .
$$

Let us now consider a variation principle with the action

$$
S=S_{E H}+S_{G H}+S_{M}
$$

where

$$
S_{E H}=\frac{1}{2 \kappa^{2}} \int_{M} \sqrt{|g|} R d x
$$

is the Einstein-Hilbert action,

$$
S_{G H}=\frac{1}{\kappa^{2}} \int_{\partial M} \sqrt{|h|} K d y
$$

is the Gibbons-Hawking boundary term ${ }^{15}$, with $K$ the trace of the second fundamental form according to the ADM decomposition (this term is required for canceling second time derivatives in the equations of motion), and $S_{M}$ some matter term.

Let us consider here a matter term $S_{M}$ corresponding to a minimally coupled scalar field $\Phi$ with potential $U(\Phi)$. Then the variational principle of (6.8) is equivalent to a Lagrangian variational principle over the minisuperspace $\mathcal{M}$ and the scalar field $\Phi$,

$$
S=\int L d t,
$$




$$
\begin{aligned}
L & =\frac{1}{2} \mu e^{-\gamma+\sum_{i=1}^{n} d_{i} \beta^{i}}\left\{\sum_{i=1}^{n} d_{i}\left(\dot{\beta}^{i}\right)^{2}-\left[\sum_{i=1}^{n} d_{i} \dot{\beta}^{i}\right]^{2}+\kappa^{2} \dot{\Phi}^{2}\right\} \\
& +\frac{1}{2} \mu e^{\gamma+\sum_{i=1}^{n} d_{i} \beta^{i}} R^{(i)} e^{-2 \beta^{1}}-\mu \kappa^{2} e^{\gamma+\sum_{i=1}^{n} d_{i} \beta^{i}} U(\Phi),
\end{aligned}
$$

where

$$
\mu:=\kappa^{-2} \prod_{i=1}^{n} \sqrt{\left|\operatorname{det} g^{(i)}\right|} .
$$

It is a convenient proceedure of cosmologists, to extend the minisuperspace $\mathcal{M}$ of pure geometry directly by an additional dimension from the scalar field $\Phi$ as further minisuperspace coordinate, yielding an enlarged minisuperspace $\mathcal{M S}:=\mathcal{M S}(M, \Phi)$.

Let us define a metric on $\mathcal{M S}$, given in coordinates $\beta^{i}, i=1, \ldots, n+1$ with $\beta^{n+1}:=\kappa \Phi$. We set

$$
G_{n+1 i}=G_{i n+1}:=\delta_{i n+1} \text { and } G_{k l}:=d_{k} \delta_{k l}-d_{k} d_{l}
$$

for $i=1, \ldots, n+1$ and $k, l=1, \ldots, n$, thus defining the components $G_{i j}$ of the minisuperspace metric

$$
G=G_{i j} d \beta^{i} \otimes d \beta^{j}
$$

Note that the signature of $\mathcal{M}$ is Lorentzian for $n>1$, and $G_{11}<0$ for $d_{1}>1$ implies that the signature of $\mathcal{M S}$ is Lorentzian not only for $n>1$ but also for $n=1$ if $d_{1}>1$. If there is at least one (e.g. compact "internal") extra factor space, i.e. $n>1$, then $\mathcal{M}$ has Lorentzian signature $(-,+, \ldots,+)$. 
After diagonalization of (6.11) by a minisuperspace coordinate transformation $\beta^{i} \rightarrow \alpha^{i}$ $(i=1, \ldots, n)$, there is just one new coordinate, say $\alpha^{1}$, which corresponds to the unique negative eigenvalue of $G$. With a further (sign preserving) coordinate rescaling, $G$ is equivalent to the Minkovsky metric. Hence $\mathcal{M}$ is conformally flat.

While $\beta^{i} \rightarrow \alpha^{i}$ is only a coordinate transformation (1) on $\mathcal{M}$ or $\mathcal{M S}$, it transforms a multidimensional geometry (2.12) with scale exponents $\beta^{i}$ to another geometry of the same multidimensional type (2.12), i.e. with the same $d_{i}$ and $d s_{i}^{2}$, but new scale exponents $\alpha^{i}$ of the factor spaces $M_{i}$. We can always perform the diagionalization of (6.11) such that $\alpha^{1}$ and hence $M_{1}$ belongs to the unique negative eigenvalue of $G$. This $M_{1}$ is identified as "external" space. The scale factors of the "internal" spaces $M_{2}, \ldots, M_{n}$ and $\Phi$ contribute only positive eigenvalues of $\mathcal{M}$ reps. $\mathcal{M S}$. (For $n=1$ there are no "internal" spaces, but $G_{11}<0$ for $d_{1}>1$ still provides a negative eigenvalue that is distinguished at least against the additional positive eigenvalue from $\Phi$ in $\mathcal{M S}$.) $\alpha^{1}$ assumes in $\mathcal{M}$ or $\mathcal{M S}$ the role played by time in usual geometry and quantum mechanics. In this way "external" space is distinguished against the "internal" spaces, because its scale factor provides a natural "time" coordinate on $\mathcal{M}$. If in the multidimensional geometry $(2.12) M_{1}$ with $\alpha^{1}$ is strictly expanding w.r.t. time $t$, then the "minisuperspace time" $\alpha^{1}$ can be considered in the geometry $g$ as a time equivalent to $t$. So the Lorentzian structure of $\mathcal{M}$ finally provides with expanding $M_{1}$ a natural "arrow of time" ${ }^{16}$.

Now we define a minisuperspace lapse function by

$$
N:=e^{\gamma-\sum_{i=1}^{n} d_{i} \beta^{i}}
$$

and a minisuperspace potential $V=V\left(\beta^{i}\right)$ via

$$
V:=-\frac{\mu}{2}\left(\sum_{i=1}^{n} R^{(i)}-2 \kappa^{2} U(\Phi)\right) e^{-2 \beta^{i}+\gamma+\sum_{j=1}^{n} d_{j} \beta^{j}} .
$$

Then

$$
L=N\left\{\frac{\mu}{2} N^{-2} G_{i j} \dot{\beta}^{i} \dot{\beta}^{j}-V\right\}
$$

Here $\mu$ is the mass of a classical particle in minisuperspace. Note that $\mu^{2}$ is proportional to the volumes of spaces $M_{i}$, which is a purely geometrical datum on $M$ but not on $\mathcal{M}$ or $\mathcal{M S}$.

In the harmonic time gauge, the equations of motion from Eq. (6.15) are given by

$$
\mu G_{i j} \ddot{\beta}^{j}=-\frac{\partial V}{\partial \beta^{i}}
$$


with the energy constraint

$$
\frac{\mu}{2} G_{i j} \dot{\beta}^{i} \dot{\beta}^{j}+V=0 .
$$

In the following we should forget, whether some of the coordinates $\beta^{i}$ are of nongeometric origin, since all will be treated equally. Therefore we will understand by $\mathcal{M}$ some minisuperspace (no matter whether it it has actually been constructed as some $\mathcal{M S}$ or not).

Canonical quantization has been considered e.g. in Refs. 10, 17 and 18. It essentially consists in replacing the constraint equation (6.17) by the WdW equation

$$
\left(-\frac{1}{2}[\Delta+a R]+V\right) \Psi=0
$$

where $\Psi$ is a wave function from a distribution space $\mathcal{S}^{*}$, which is the dual of the test function space $\mathcal{S} \subset \mathcal{H}$, dense in the Hilbert space $\mathcal{H}=\mathcal{H}(\mathcal{M})$. (Often one might think of $\mathcal{S}$ as the Schwartz space and correspondingly of $\mathcal{S}^{*}$ as the space of tempered distributions over $\mathcal{S}$. Note however that the proper choice of $\mathcal{S}$ depends on the Hamiltonian $H$, and more specifically on the shape of the potential $V$.)

The Lagrangian (6.15) is invariant under arbitrary time reparametrization $h \in$ Diff $\mathbb{R}$ acting via

$$
h\left(\beta^{i}\right)(t):=\beta^{i}(h(t)) \quad \text { and } \quad h(N)(t):=N(h(t)) \frac{d h}{d t}
$$

on minisuperspace coordinates $\beta^{i}$ and the lapse function $N$.

We set in the following

$$
N=: e^{-2 f}
$$

and admit $f \in C^{\infty}(\mathcal{M})$ to be an arbitrary smooth function on $\mathcal{M}$.

In the time gauge given by $f$ the Lagrangian is

$$
L^{f}:=\frac{\mu}{2}_{f} G_{i j}(\beta) \dot{\beta}^{i} \dot{\beta}^{j}-V^{f}(\beta)
$$

and the energy constraint is

$$
E^{f}:=\frac{\mu_{f}}{2} G_{i j}(\beta) \dot{\beta}^{i} \dot{\beta}^{j}+V^{f}(\beta)=0,
$$

where

$$
{ }^{f} G=e^{2 f} G \text { and } V^{f}=e^{-2 f} V .
$$

With canonical momenta

$$
\pi_{i}=\frac{\partial L^{f}}{\partial \dot{\beta}^{i}}=\mu G_{i j}^{f} \dot{\beta}^{j}
$$


this is equivalent to the Hamiltonian system given by

$$
H^{f}=\frac{1}{2 \mu}\left({ }^{f} G\right)^{i j} \pi_{i} \pi_{j}+V^{f}
$$

and the energy constraint

$$
H^{f}=0
$$

There the inverse of the minisuperspace metric is given by ${ }^{f} G^{-1}=e^{-2 f} G^{-1}$, where for the system with Eq. (6.11) the components of $G^{-1}$ are

$$
G^{i j}=\frac{\delta_{i j}}{d_{i}}+\frac{1}{1-\sum_{i=1}^{n} d_{i}} .
$$

At quantum level $H^{f}$ has to be replaced by an operator $\hat{H}^{f}$, acting by the energy constraint

$$
\hat{H}^{f} \Psi^{f}=0
$$

on $\Psi^{f} \in \mathcal{S}^{* f}$, where $\mathcal{S}^{* f}$ is given by the action of a represention $D_{b}$ of $C^{\infty}(\mathcal{M})$ with conformal weight $b$ on $\Psi \in \mathcal{S}^{*}$, i.e. for $f \in C^{\infty}(\mathcal{M})$

$$
\Psi^{f}=D^{b}(f)(\Psi)=e^{b f} \Psi
$$

Note that correspondingly a testfunction $\varphi \in \mathcal{S}$ has to transform to $\varphi^{f}=e^{-b f} \varphi \in \mathcal{S}^{f}$ in order to keep $\Psi[\varphi]$ conformally invariant. Generally on a dual space the weight should be the negative of the weight on the original space. In our application to the quantization of $H^{f}$ from (6.23), the condition

$$
\hat{H}^{f}=e^{-2 f} e^{b f} \hat{H} e^{-b f}
$$

implies that

$$
\hat{H}^{f}=-\frac{1}{2 \mu}\left[\Delta^{f}-\xi_{c} R^{f}\right]+V^{f}
$$

on wavefunctions $\Psi^{f}=e^{b f} \Psi \in \mathcal{S}^{* f}$. The WdW equation (6.26) is conformally equivariant if and only if Eq. (6.26) for any $f$ is equivalent to

$$
\hat{H} \Psi=0
$$

where

$$
\hat{H}=\left.\hat{H}^{f}\right|_{f=0} \text { and } \Psi=\left.\Psi^{f}\right|_{f=0}
$$

are Hamilton operator and wavefunction in the harmonic time gauge. 


\section{The Number $\xi_{c}$ in Different Dimensions}

In Sec. 4 and 5 we have already seen that, among all possible values for the coupling constants $\xi \equiv-a$, only $\xi=\xi_{c}$ allows a conformal representation of both, the classical and the quantum theory. According to the examples of Sec. $4, \xi=\xi_{c}$ is also a critical value, where the relationship between the scalarfields of two conformally related Lagrangian models, one with minimal the other with non-minimal coupling, changes qualitatively.

In this section we examine the dependence of this critical number $\xi_{c}=\frac{D-2}{4(D-1)}$ on the dimension $D$. Therefore we consider the prime factorization of $\xi_{c}$. Table 1 lists $\xi_{c}=: \frac{r}{s}$ with trivial greatest common divisor of $r$ and $s$, i.e. $\operatorname{gcd}(r, s)=1$, the maximal primefactor $p_{m}$ contained in either $r$ or $s$, and the least common multiple $\operatorname{lcm}\left(\xi_{c}\right):=\operatorname{lcm}(r, s)$, for dimensions $D=3 \ldots 30$.

$\begin{array}{ccccccccccccccccc}D & : & 3 & 4 & 5 & 6 & 7 & 8 & 9 & 10 & 11 & 12 & 13 & 14 & 15 & 16 \\ \xi_{c}=\frac{r}{s} & : & \frac{1}{8} & \frac{1}{6} & \frac{3}{16} & \frac{1}{5} & \frac{5}{24} & \frac{3}{14} & \frac{7}{32} & \frac{2}{9} & \frac{9}{40} & \frac{5}{22} & \frac{11}{48} & \frac{3}{13} & \frac{13}{56} & \frac{7}{30} \\ p_{m} & : & 2 & 3 & 3 & 5 & 5 & 7 & 7 & 3 & 5 & 11 & 11 & 13 & 13 & 7 \\ l \mathrm{lcm} & : & 8 & 6 & 48 & 5 & 120 & 42 & 224 & 18 & 360 & 110 & 528 & 39 & 728 & 210 \\ 17 & 18 & 19 & 20 & 21 & 22 & 23 & 24 & 25 & 26 & 27 & 28 & 29 & 30 \\ \frac{15}{64} & \frac{4}{17} & \frac{17}{72} & \frac{9}{38} & \frac{19}{80} & \frac{5}{21} & \frac{21}{88} & \frac{11}{46} & \frac{23}{96} & \frac{6}{25} & \frac{25}{104} & \frac{13}{54} & \frac{27}{112} & \frac{7}{29} \\ 5 & 17 & 17 & 19 & 19 & 7 & 11 & 23 & 23 & 5 & 13 & 13 & 7 & 29 \\ 960 & 68 & 1224 & 342 & 1520 & 105 & 1848 & 506 & 2208 & 150 & 2600 & 702 & 3024 & 203\end{array}$

Table 1: $p_{m}$ and $\mathrm{lcm}$ of $\xi_{c}$ for $D=3, \ldots, 30$.

We see: The smaller $\operatorname{lcm}\left(\xi_{c}\right)$, the simpler $\xi_{c}$ is as a fraction. $\operatorname{lcm}\left(\xi_{c}\right)$ has its lowest value for $D=6$, followed by $D=4$ and $D=3$. In these dimensions $\xi_{c}^{-1}$ is just an integer. Besides $D=3,4$ and 6 , the next best is $D=10$ with $\xi_{c}=\frac{2}{9}$.

Note that in any dimension $D=4 i+2, i \in \mathbb{N}, \operatorname{lcm}\left(\xi_{c}\right)$ is lower than for $D-2, D-1$ and $D+1$. When we admit for the rational composition only the first 3 prime numbers and consider only dimensions $D$ in which $p_{m} \leq 5$, then the lowest value of $\operatorname{lcm}\left(\xi_{c}\right)$ for $D>10$ is $D=26$.

The special simplicity of $\xi_{c}$ in dimensions $D=3,4,6$ and 10 might be related to an interplay between conformal symmetry breaking in subspaces of the universe and the selection of its dimensions. Resonances of fundamental frequencies coupled by $\xi_{c}$ might induce dimensional reduction or compactification. Unfortunately a sufficient explanation is presently not at hand. 


\section{Conclusion}

We have emphasized that conformal coordinate transformations (1) have to be distinguished sharply from conformal transformations of the geometry (2), especially in Lagrangian models. Similarily conformal equivalence transformations (2) of the classical Lagrangian models and minisuperspace conformal transformations (3) are conceptually very different proceedures, which have to be kept apart very carefully. An active conformal transformation (2) of a pure $D$-dimensional geometry yields a coordinate transformation on the (mini-)superspace geometry. For multidimensional geometries (2.12) conformal transformations of the factorspaces lead just to translations in the corresponding coordinates on minisuperspace $\mathcal{M}$.

Using invariance under (1) we have initially compared natural time gauges in multidimensional universes: (i) synchronous time, (ii) conformal times of different factor spaces, (iii) mean conformal time and (iv) harmonic time. Transitions between them are given by special conformal coordinate transformations.

As an example for invariance under (2) the conformal transformation between the minimal coupling model and the conformal coupling model has been performed in arbitrary dimensions $D$. The obtained conformal factor and scalar field are in agreement with the result of Ref. 13.

By Eq. (4.26) the generalization of the scalar field from the conformal coupling case to that of arbitrary coupling $\xi$ has been found in arbitrary dimension $D$. For $D=4$ this expression corresponds to the earlier result of expressions Eqs. (3.3-5) in Ref. 7 qualitatively. (Note also that Eq. (5) in Ref. 8 holds only with $\xi=\xi_{c}=\frac{1}{6}$.)

At $\phi^{2}=\xi_{c}^{-1}$ there is a singularity of the conformal transformation. Hence the conformal equivalence only holds separately in the ranges $\phi^{2}<\xi_{c}^{-1}$ and $\phi^{2}>\xi_{c}^{-1}$.

It is a characteristic feature that natural time gauges (1) are not preserved under conformal transformation (2) of geometry.

Similarily we have no reason to expect that classically equivalent conformal models (2) could by canonical quantization have minisuperspace conformal WdW equations equivalent under (3). This is specially evident, when the minisuperspace containes also data beyond pure geometry, e.g. a scalar field. While a scalar field coupled to $D$-dimensional geometry transforms to the scalar field of the equivalent model by a complicated integral transform (see e.g. Eq. (4.26)), on minisuperspace $\mathcal{M S}$ it is just described as an additional 
coordinate, on equal footing with those from the scale factors of geometry. Hence, from the conceptual point of view, the attempt to treat a tensor field (the geometry) and a scalar field on a common geometrical footing might be questionabel in the context of canonical (minisuperspace) quantization. For a pure geometry however minisuperspace can be understood better, at least for the multidimensional geometry (2.12).

Nevertheless it remains an interesting question for further investigations to find out, how solutions of the WdW equation corresponding to equivalent solutions of equivalent Lagrangian models are related. Interesting solutions of the WdW equation for a homogeneous scalar field minimally coupled to multidimensional geometry have been obtained in Refs. 1, 3, 19 and 20.

The minisuperspace $\mathcal{M}$ for a pure geometry with nontrivial "internal" factor spaces $M_{2}, \ldots, M_{n}$, or $\mathcal{M S}$ for geometry coupled to a scalar field, is conformally Minkovskian. The negative eigenvalue of its metric $G$ can be associated with an expanding "external" factor space $M_{1}$ yielding a natural cosmological "arrow of time" (see Ref. 16).

Finally in Sec. 7 we have seen, that besides playing a distinguished role for invariance under both (2) and (3), the conformal coupling $\xi_{c}=\frac{D-2}{4(D-1)}$ indicates number theoretically distinguished dimensions $D=3,4,6$ and 10 , which are (besides $D=1,2$ ) the most important subspace dimensions appearing in realistic models of the universe. Further investigations will have to find a satisfactory explanation of this coincidence.

\section{Acknowledgements}

Support by DFG grant Bl 365/1-1 is gratefully acknowledged. The author thanks both, the Projektgruppe Kosmologie at Universität Potsdam, especially H.-J. Schmidt, and the Gravitationsprojekt with U. Bleyer, for their hospitality and support. Thanks for critical comments go to U. Bleyer, H.-J. Schmidt and A. Zhuk. 


\section{References}

${ }^{1}$ U. Bleyer, M. Rainer and A. Zhuk, Conformal Transformation of Multidimensional Cosmological Models and their Solutions, Preprint FUB-HEP/94-3, FU Berlin (1994).

${ }^{2}$ U. Bleyer, Multidimensional Cosmology, p. 101-11 in: The Earth and the Universe (A Festschrift in Honour of Hans-Jürgen Treder), ed.: W. Schröder, Science Ed., Bremen (1993).

${ }^{3}$ V. D. Ivashchuk, V. N. Melnikov, A. I. Zhuk, Nuovo Cim. B 104, 575 (1989).

${ }^{4}$ H. J. Schmidt, The Metric in the Superspace of Riemannian Metrics and its Relation to Gravity, p. 405 in: Diff. Geom. and Appl., ed.: D. Krupka (Brno 1989).

${ }^{5}$ S. T. Swift, J. Math. Phys. 33, 3723 (1992); J. Math. Phys. 34, 3825 (1993); J. Math. Phys. 34, 3841 (1993).

${ }^{6}$ S. Gottlöber, V. Müller, H.-J. Schmidt and A. A. Starobinsky, Int. J. Mod. Phys. D 1, 257 (1992).

7 T. Futamase and K. Maeda, Phys. Rev. D 39, 399 (1989).

8 D. N. Page, J. Math. Phys. 32, 3427 (1991).

${ }^{9}$ H. J. Schmidt, Phys. Lett. B 214, 519 (1988).

10 J. J. Halliwell, Phys. Rev. D 38, 2268 (1988).

${ }^{11}$ M. C. Gutzwiller, Chaos in Classical and Quantum Mechanics, p. 197, Series: Interdisciplinary Applied Mathematics 1, Springer-Verlag, New York (1988).

${ }^{12}$ K. Maeda, Phys. Rev. D 39, 3159 (1989).

${ }^{13}$ B. C. Xanthapoulos and Th. E. Dialynas, J. Math. Phys. 33, 1463 (1992).

${ }^{14}$ D. V. Gal'tsov and B. C. Xanthopoulos, J. Math. Phys. 33, 273 (1992).

${ }^{15}$ G. W. Gibbons and S. W. Hawking, Phys. Rev. D 15, 2752 (1977).

${ }^{16}$ H. D. Zeh, The Physical Basis of the Direction of Time, 2nd. ed. Springer-Verlag (Heidelberg, 1991).

17 T. Christodoulakis and J. Zanelli, Nuovo Cim. B 93, 1 (1986).

18 T. Christodoulakis and J. Zanelli, Class. Quantum Grav. 4, 851 (1987).

${ }^{19}$ A. I. Zhuk, Class. Quant. Grav. 9, 2029 (1992).

${ }^{20}$ A. I. Zhuk, Sov J. Nucl. Phys. 55, 149 (1992); Phys. Rev. D 45, 1192 (1992). 